\title{
The Construct of the Schizophrenia Quality of Life Scale Revision 4 for the Population of Taiwan
}

\author{
Chia-Ting Su, ${ }^{1}$ Ai-Lun Yang, ${ }^{2}$ and Chung-Ying Lin $^{3}$ \\ ${ }^{1}$ Department of Occupational Therapy, College of Medicine, Fu Jen Catholic University, New Taipei City, Taiwan \\ ${ }^{2}$ Department of Sports Sciences, University of Taipei, Taipei, Taiwan \\ ${ }^{3}$ Department of Rehabilitation Sciences, Faculty of Health and Social Sciences, The Hong Kong Polytechnic University, \\ 11 Yuk Choi Road, Hung Hom, Hong Kong
}

Correspondence should be addressed to Chung-Ying Lin; cylin36933@gmail.com

Received 22 July 2016; Accepted 15 January 2017; Published 31 January 2017

Academic Editor: Erna I. Blanche

Copyright (C) 2017 Chia-Ting Su et al. This is an open access article distributed under the Creative Commons Attribution License, which permits unrestricted use, distribution, and reproduction in any medium, provided the original work is properly cited.

This study examines the factor structure of the Schizophrenia Quality of Life Scale Revision 4 (SQLS-R4) for inpatients with schizophrenia in a psychiatric hospital in southern Taiwan. All the participants $(n=100)$ filled out the SQLS-R4, Mini Mental Status Examination (MMSE), and Brief Psychiatric Rating Scale (BPRS) under the supervision of one experienced occupational therapist. Using confirmatory factor analysis, we first determined that a 29-item model was more satisfactory than the original 33-item model based on the findings of better fit indices for the 29-item model. We then found that a three-correlated-factor structure was best for the SQLS-R4 after four models (namely, two-correlated-factor, three-correlated-factor, seven-correlated-factor, and second-order models) had been compared. In addition, the three constructs (psychosocial, physical, and vitality) were moderately to highly correlated with the constructs of the World Health Organization Quality of Life- (WHOQOL-) BREF ( $r=-0.38$ to -0.69 ), except for one low correlation between the vitality construct of the SQLS-R4 and the psychological construct of the WHOQOL-BREF $(r=-0.26)$. We tentatively conclude that the SQLS-R4 with a three-correlated-factor structure is a valid and reliable instrument for examining the quality of life of people with schizophrenia.

\section{Introduction}

Quality of life (QoL) measures have become more important for therapeutic interventions and clinical decisions $[1,2]$, especially for patients with chronic illness [3]. When people with schizophrenia confront their chronic disabling illness, it is important for healthcare professionals to understand their QoL. QoL, a subjective perception of an individual's health position within their cultural context and value system [4], can be used as one of the long-term goals of medical interventions [3].

Two kinds of QoL measures can be applied to people with schizophrenia: generic QoL measures and schizophreniaspecific QoL measures [5]. Although the schizophreniaspecific QoL measures cannot compare QoLs between different populations with disabilities (e.g., people with stroke and people with spinal cord injury), they focus on the core symptoms that people with schizophrenia encounter
[5]. Therefore, the schizophrenia-specific QoL measures are particularly useful for understanding how the symptoms affect the QoL of people with schizophrenia.

Several schizophrenia-specific QoL measures have been developed, and the Schizophrenia Quality of Life Scale Revision 4 (SQLS-R4) has been suggested to be one of the most useful ones [5-7]. In addition to its items specifically designed for people with schizophrenia $[5,8]$, its other strengths include (1) practical and short content to fill out (10-15 minutes to complete); (2) improved psychometric properties after several revisions [6,9]; (3) solid factor structure and internal reliability [7-12]; and (4) availability of cross-cultural comparisons by providing rigorously translated versions in 52 languages through standardized procedures: forward translation, reconciliation, and back translation [9].

However, as a newly developed QoL measure $[8,11]$, the construct of SQLS-R4 still needs to be confirmed. Although the SQLS-R4 has good psychometric properties $[6,10,13]$, to 
the best of our knowledge, only one UK study [7] has used confirmatory factor analysis (CFA) to examine the construct of SQLS-R4. The advantage of using CFA is being able to compare several proposed models and to clarify the construct of the tested instruments [14]. Therefore, the CFA is suitable for understanding the constructs of measurements that are under development, such as the SQLS-R4. Although the SQLS-R4 was suggested to be two-dimensional $[6,7,12,13]$, Martin and Allan [7] indicated that some CFA fit indices are not acceptable (root mean square error of approximation [RMSEA] $=0.11$ and weighted root mean square residual [WRMR] $=0.94$ ). In addition, one Singaporean study [11] mentions that the cultural difference between Asia and the West may influence the psychometric properties of the SQLS-R4. Thus, the construct of the SQLS-R4 may differ in Asian and Western cultures. Therefore, for better application and interpretation of the SQLS-R4, we suggest that additional studies on the SQLS-R4 construct in Asian populations are needed.

The former version of the SQLS-R4, SQLS, is a 30item self-reported measure compromising three domains (psychosocial, motivation and energy, and symptoms and side effects) [8]. Several revisions were carried out for the SQLS to improve its psychometric properties, and finally the SQLS-R4 with two factors was developed with different language versions [7, 9], including Taiwanese version $[6,13]$. However, when Kuo et al. [6] examined the psychometric properties of the Taiwan version of SQLS-R4, they found that four items had weak corrected item-total correlation with their domains. Therefore, Kuo et al. [13] further removed the four items and used the retained items to do the exploratory factor analysis. They found that the Taiwan version of SQLS$\mathrm{R} 4$ contained seven factors and proposed three dimensions (psychosocial, physical condition, and validity) to include the seven factors. Kuo et al. [13] proposed the physical condition as one of the dimensions because physical domain is a key component for QoL. Hence, current literature suggests different factor structures for the SQLS-R4 according to its item numbers (29 or 33 items) and underlying factors (2, 3, and 7 factors/dimensions).

Five CFA models of the SQLS-R4 construct are proposed in our study. In addition to the two-factor model, we propose three more models based on the findings and suggestions of one Taiwanese study [13]: a three-factor model (psychosocial, physical, and vitality), a seven-factor model (relationships with others, loneliness, depressed thinking, worry, exhaustion status, somatic concern, and vitality), and a second-order model with seven first-order factors (relationships with others, loneliness, depressed thinking, worry, exhaustion status, somatic concern, and vitality) embedded in three secondorder factors (psychosocial, physical, and vitality). Moreover, four items have been found to be weak for the SQLS-R4 Taiwan version [13], and, thus, a comparison between a 33item model and a 29-item model was conducted.

We investigated the SQLS-R4 construct for the population of Taiwan and suggest appropriate items to improve the SQLS-R4's psychometric properties. Specifically, we first compared 33-item, 29-item, three-factor, seven-factor, and second-order models. We then tested the determined underlying constructs for their concurrent validity with one generic
QoL measure (World Health Organization Quality of Life [WHOQOL]-BREF).

\section{Methods}

The study was approved by the Institutional Review Board of National Cheng Kung University Hospital, and all the participants filled out and signed informed consents before beginning this study.

2.1. Participants and Procedure. Convenience sampling was used to recruit the participants from a psychiatric hospital in southern Taiwan. Like some other mental institutions in Taiwan, this psychiatric hospital was mainly designed to take long-term care of patients with chronic mental illness. Although symptoms remained relatively stable, most of the patients in this hospital were unable to totally live independently in community due to severity of symptoms, insufficient family support, or other issues. All the participants in our study were diagnosed with schizophrenia based on the DSM IV-TR [15] definition, and all were more than 18 years old (age range: 27 to 66). In addition, they all met the inclusion criteria of an illness duration $>2$ years, no medication adjustment within the previous two months, a score $\geq 24$ on the Mini Mental Status Examination (MMSE), and a score $<24$ on the Brief Psychiatric Rating Scale (BPRS). Participants were excluded if they met the following exclusion criteria: (1) impaired cognitive ability to complete the questionnaires as assessed by one occupational therapist with more than 10 years of clinical experience using clinical observation and (2) with comorbid diagnoses of anxiety, depression, bipolar disorder, organic mental disorder, dementia, intellectual disability, and learning disability. All participants were asked to complete several questionnaires mentioned below. Finally, the data of 100 participants, a minimum criterion for performing CFA $[16,17]$, were used in the study.

\subsection{Instruments}

2.2.1. The Schizophrenia Quality of Life Scale Revision 4 (SQLSR4). The SQLS was initially developed to resolve the lack of QoL measures for people with schizophrenia [8]. The original version of the SQLS contains 30 items distributed between three factors: psychosocial (15 items), motivation and vitality (7 items), and symptoms and side effects ( 8 items). It has a satisfactory internal consistency ( $\alpha=0.80-0.93$ ) [8]. After several revisions that improved its psychometric properties, the latest version of the SQLS (SQLS-R4) contains 33 items distributed between two factors: psychosocial (20 items) and vitality (13 items) [9]. The SQLS-R4 is self-rated, and all but four items are coded on a 5-point scale in relation to their frequency of occurrence during the previous week: $0=$ never and $4=$ always. The exceptional 4 items are coded the opposite way: $0=$ always and $4=$ never. A higher SQLS-R 4 score represents a worse QoL.

2.2.2. The WHO Questionnaire on the Quality of Life, Short Form (WHOQOL-BREF). We used the WHOQOL-BREF Taiwan version, which has 28 items (two items are domestic 
items, and other items are international items). Four domains (physical health, psychological health, social relations, and environment) are included in the WHOQOL-BREF. The psychometric properties of the WHOQOL-BREF Taiwan version have been tested (e.g., the internal consistency [Cronbach's $\alpha=0.70-0.91]$, the test-retest reliability $[r=0.76-0.80]$, and the construct validity $[\mathrm{CFI}=0.89]$ ) and were found to be satisfactory [18]. In addition, a recent study found that the construct of the WHOQOL-BREF Taiwan version fit well with the schizophrenia population and is suitable for using to measure the QoL of people with schizophrenia [19]. A higher WHOQOL-BREF score represents a better QoL.

2.2.3. Mini Mental State Examination (MMSE). The MMSE contains 11 items and has a total score of 30 . It is commonly used to screen the function of cognition; a score $\geq 24$ suggests an intact cognition function. The test-retest reliability $(r=$ $0.89-0.98)$ and interrater reliability $(r=0.83)$ have been examined for the Chinese version of the MMSE [20].

2.2.4. Brief Psychiatric Rating Scale (BPRS). The BPRS contains 16 items; it is designed to measure the severity of psychiatric symptoms. A lower BPRS score indicates a better psychological condition, and the internal consistency of the Chinese version of BPRS is good (Cronbach's $\alpha=0.80$ ) [21].

2.3. Data Analysis. The construct of the SQLS-R4 was examined using CFA models with maximum-likelihood estimations. Five CFA models were tested: Models 1 (33 items; Figure 1) and 2 (29 items; Figure 2) were two-correlatedfactor models (psychosocial and vitality). Models 3 and 4 were three-correlated-factor (psychosocial, physical, and vitality; Figure 3) and seven-correlated factor (relationships with others, loneliness, depressed thinking, worry, exhaustion status, somatic concern, and vitality; Figure 4) models, respectively. Unlike Models 1 to 4, all first-order models, Model 5 (Figure 5) was a second-order model with three dimensions correlated in the second-order (psychosocial, physical, and vitality), and seven first-order factors embedded in the second-order factors (psychosocial: relationships with others, loneliness, depressed thinking, and worry; physical: exhaustion status and somatic concern; and vitality: vitality). The underlying factors of the proposed models were correlated based on the suggestions of Martin and Allan [7].

To examine the data-model fit for the five models, we used the $\chi^{2}$ test and four additional indices: the comparative fit index (CFI), Tucker-Lewis index (TLI), root mean square error of approximation (RMSEA), and standardized root mean square residual (SRMR). The values of the CFI and TLI were $>0.9$ [22], and those of the RMSEA and SRMR were $<0.08$, which suggests that the data-model fit is acceptable $[23,24]$. Moreover, to compare the five models, Akaike's information criterion (AIC) and expected cross-validation index (ECVI) were consulted; smaller values indicate a better fit [24].

After the construct of the SQLS-R4 had been determined, concurrent validity using Pearson correlation was done to strengthen the validity performance of the SQLS-R4. The correlation between each domain of the WHOQOL-BREF
TABLE 1: Participants' characteristics.

\begin{tabular}{lc}
\hline Variable & $n$ or mean \pm \\
\hline Gender & SD \\
$\quad$ Male & 66 \\
$\quad$ Female & 34 \\
Education level & \\
$\quad$ Junior high school or below & 44 \\
$\quad$ Senior high school or above & 56 \\
Previously or currently employed? & \\
$\quad$ Yes & 39 \\
$\quad$ No & 61 \\
Marital status & \\
$\quad$ Single & 76 \\
$\quad$ Married & 10 \\
$\quad$ Other & 14 \\
Age (year) & $49.2 \pm 7.8$ \\
Age at onset of schizophrenia (year) & $22.6 \pm 5.8$ \\
Duration of schizophrenia (year) & $26.6 \pm 8.1$ \\
Duration of institutionalization for & $17.4 \pm 8.7$ \\
schizophrenia (year) &
\end{tabular}

and each factor of the SQLS-R4 was tested, and an absolute correlation coefficient $>0.3$ suggests a fair correlation [25].

The CFAs were done using Lisrel 8.8 (Scientific Software International, Lincolnwood, IL, USA), and Pearson correlation was done using SPSS 16.0 (SPSS Inc., Chicago, IL, USA).

\section{Results}

The age, age at onset of schizophrenia, duration of schizophrenia, and duration of institutionalization for schizophrenia of the participants are presented in Table 1. Two-thirds of the participants were male, sixty-one percent of them had never been employed, and more than three-quarters of them were single (Table 1).

For the model comparisons, we first determined that the 29-item SQLS-R4 (Model 2: AIC = 685.519; ECVI = 6.924) was a better data-model fit than was the 33-item SQLS-R4 (Model 1: AIC $=994.108 ;$ ECVI $=10.041)$ (Table 2). Thus, the remainder of the models examined (3, 4, and 5) had 29 items. All of Model 3's fit indices were acceptable (CFI $=0.967$, TLI $=0.964, \mathrm{RMSEA}=0.068, \mathrm{SRMR}=0.072, \mathrm{AIC}=666.993$, and $\mathrm{ECVI}=6.737)$ and outperformed the other models $(\mathrm{CFI}=$ 0.928 to 0.963 , $\mathrm{TLI}=0.918$ to $0.960, \mathrm{RMSEA}=0.072$ to 0.107 , $\mathrm{SRMR}=0.073$ to 0.132 , $\mathrm{AIC}=685.519$ to 914.583 , and $\mathrm{ECVI}=$ 6.924 to 9.238). Based on the results, the SQLS-R4 fit the firstorder three-dimensional construct the best. In addition, the standardized factor loadings of Model 3 were all significant (Table 3).

The correlation between the SQLS-R4 and the WHOQOL$\mathrm{BREF}$ was then examined. All the factors underlying the SQLS-R4 were significantly and fairly correlated with the dimensions in the WHOQOL-BREF (all $P$ s $<0.01$ and absolute $r>0.3$ ) except for the correlation between vitality and psychological $(r=-0.26, P<0.01)$ (Table 4$)$. 


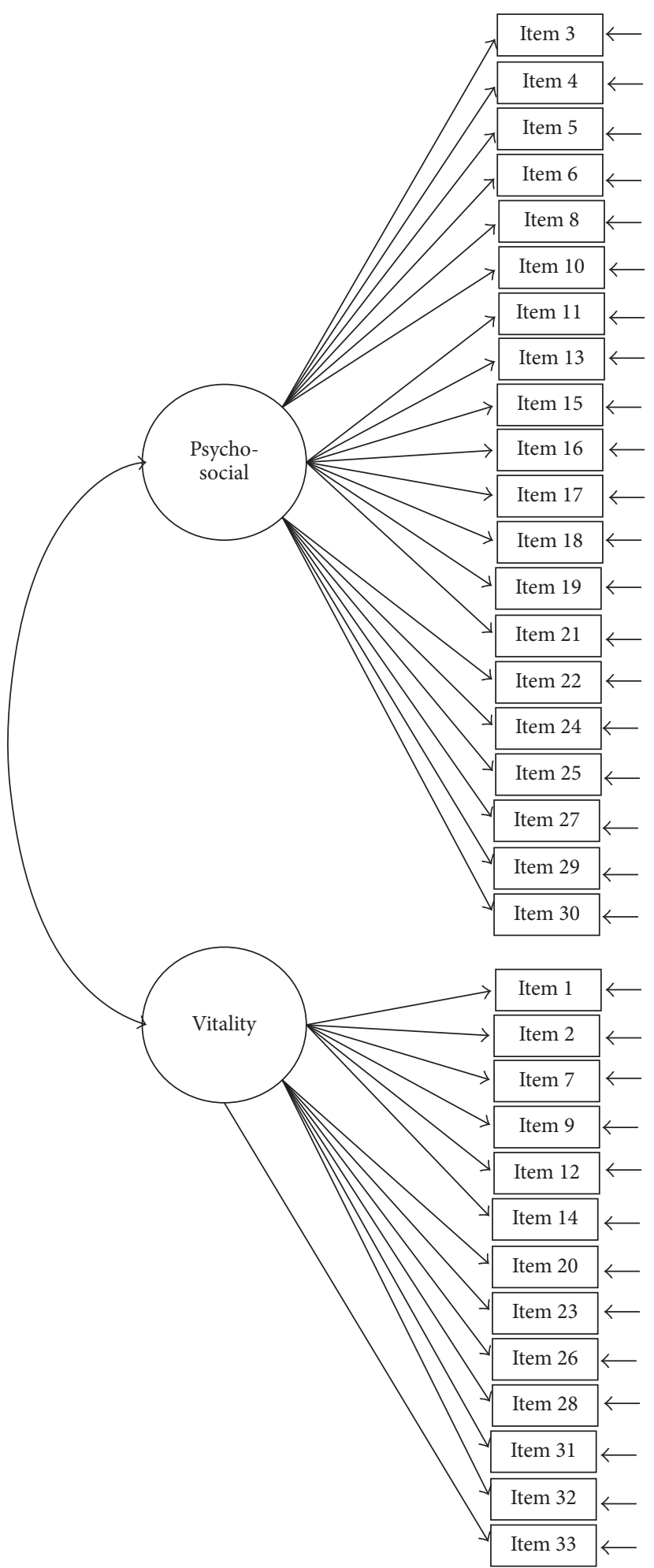

FIGURE 1: Model 1: the two-correlated-factor model with 33 items. Item descriptions are in Table 3.

\section{Discussion}

In this study, we examined the construct of a newly developed, schizophrenia-specific self-reported QoL measure (SQLS-R4) for people with schizophrenia in Taiwan. To the best of our knowledge, this is the first study that compares several possible factor structures of the SQLS-R4 using CFA and that confirms the construct of the SQLS-R4 for the Asian population. The proposed three-factor model (psychosocial, physical, and vitality) fit best with our data. Moreover, this 


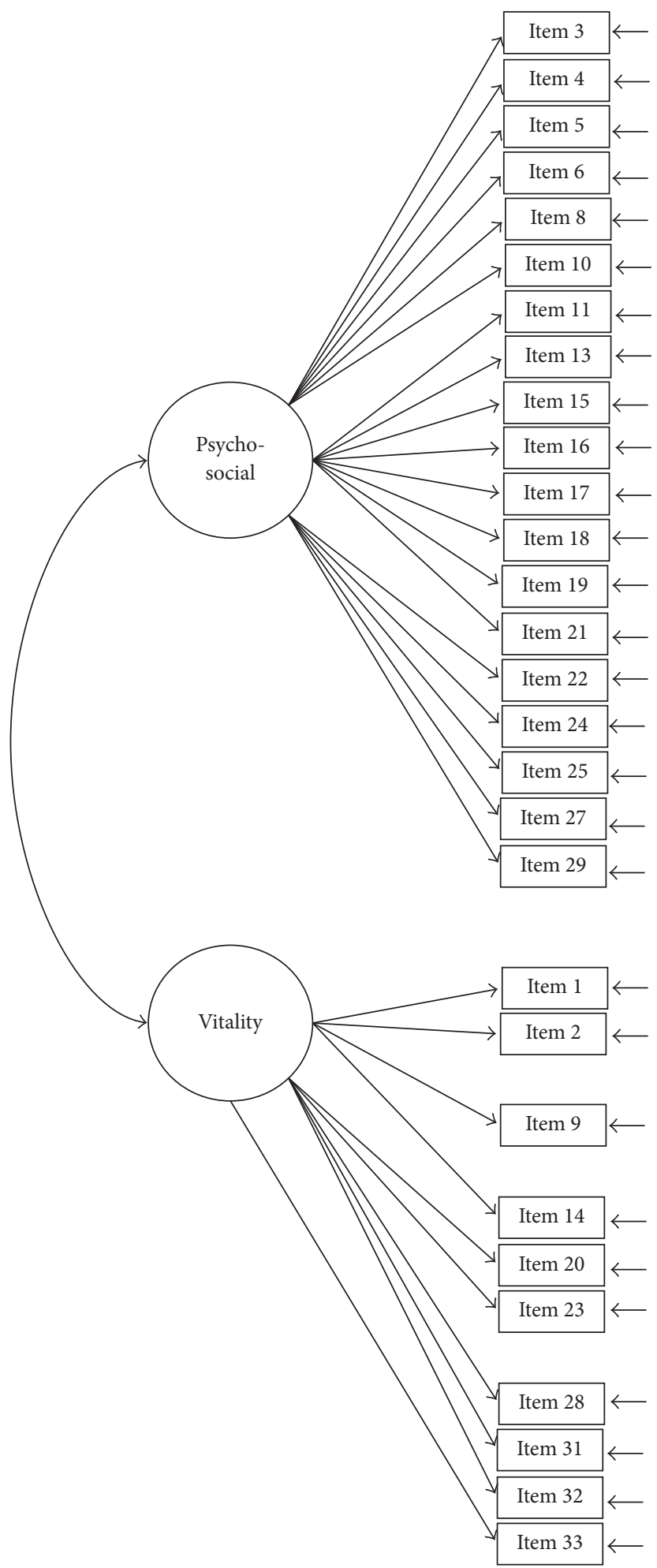

FIgURE 2: Model 2: the two-correlated-factor model with 29 items. Item descriptions are in Table 3.

proposed model was supported by the correlation between its 3 factors on the SQLS-R4 and the factors of the WHOQOLBREF.

Based on our belief that the underlying concepts should all be correlated because of their relevance to QoL, we did not examine the uncorrelated-factor models. Similar findings were concluded by Martin and Allan [7], who proposed three-factor structures (namely, a one-factor structure, a two-correlated-factor model, and a two-uncorrelated-factor model) and found that the two-correlated factor model 


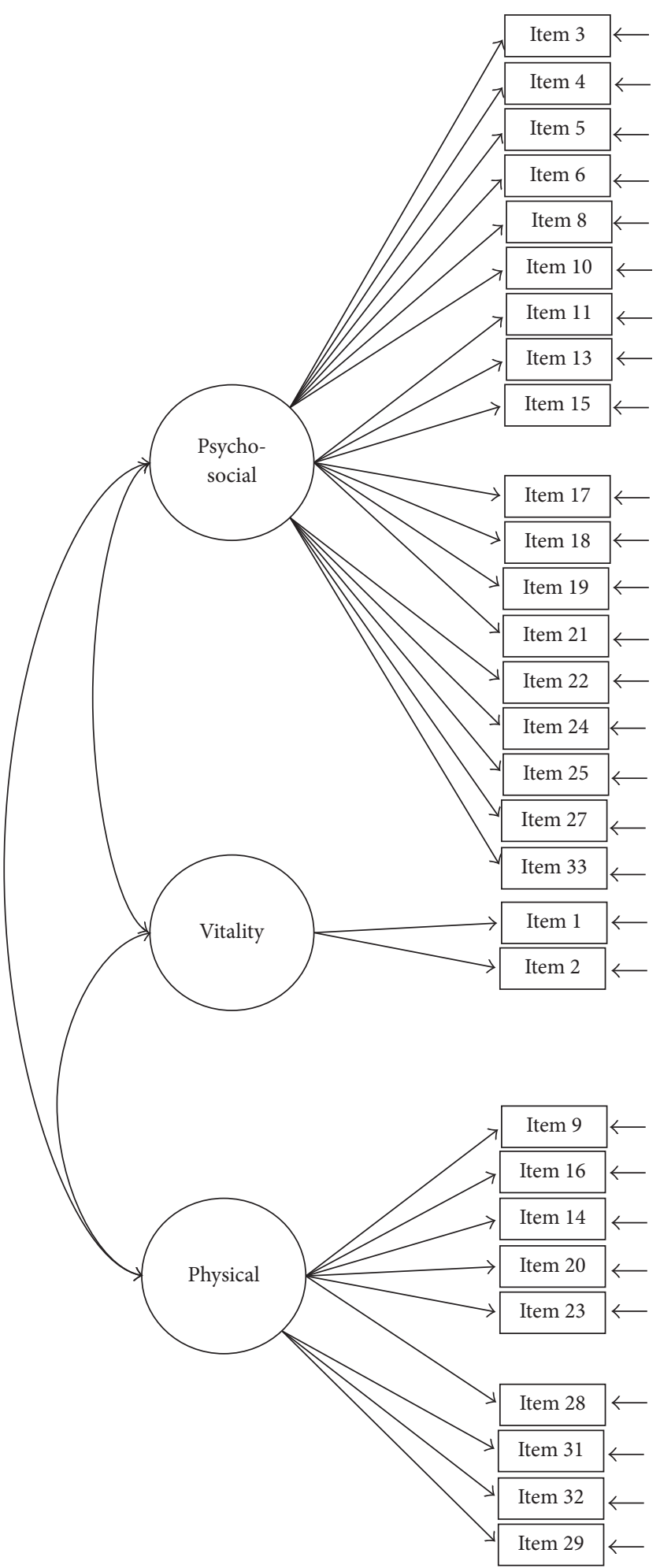

FIGURE 3: Model 3: the three-correlated-factor model with 29 items. Item descriptions are in Table 3.

had the best data-model fit. However, they also reported that the two-correlated-factor model was not good enough. Therefore, in our study, three more factor structures were compared, and we found that the three-correlated-factor model (psychosocial, physical, and vitality) was the best construct for the SQLS-R4. In addition, our results also suggested that four items on the SQLS-R4 (i.e., Item 7 [able to carry out daily activities]; Item 12 [feel I can cope]; Item 26 [feel happy]; and Item 30 [concerned about social life]) can be omitted. Items 26 and 30 are new to the SQLS-R4 


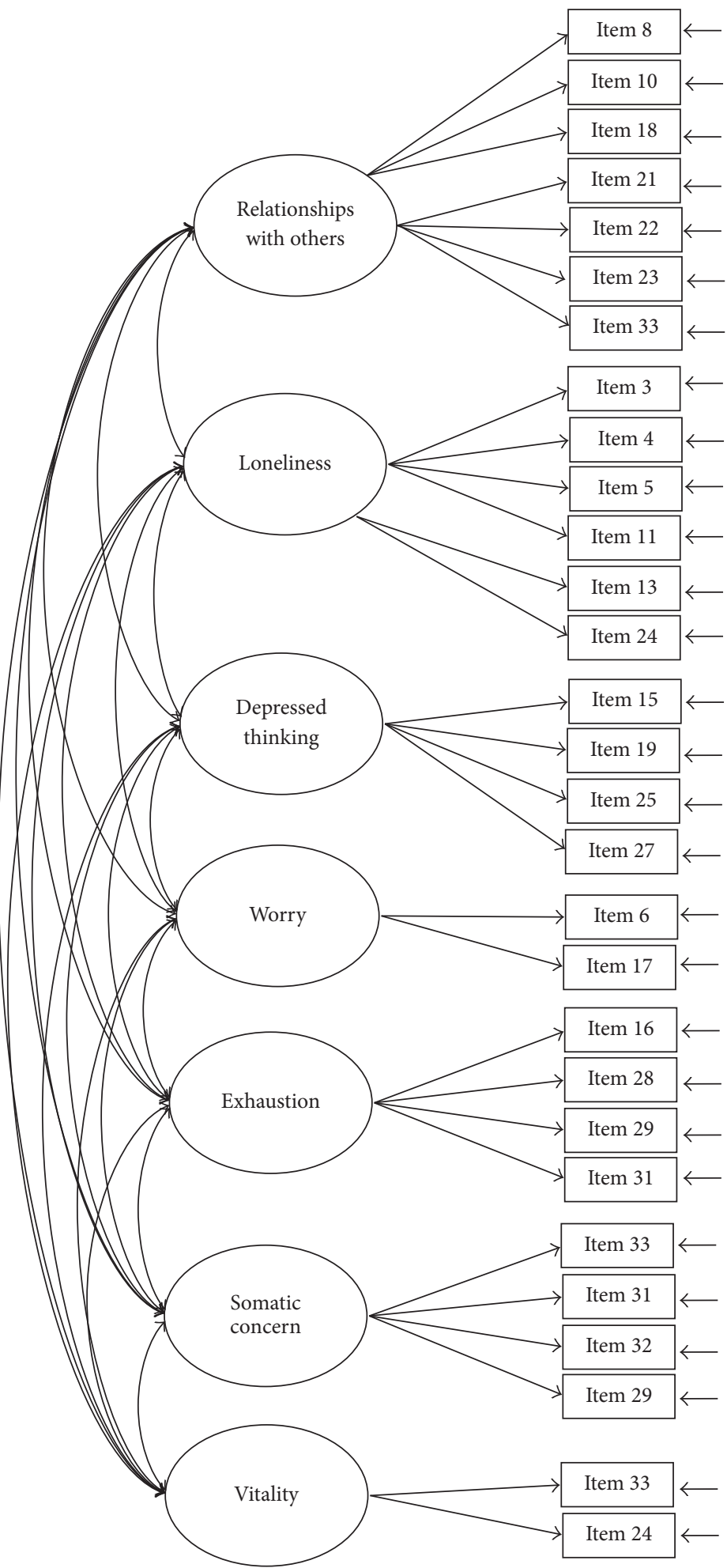

FIGURE 4: Model 4: the seven-correlated-factor model with 29 items. Item descriptions are in Table 3.

and have relatively low factor loadings $(<0.3)$ in the vitality construct and psychosocial construct, in previous studies $[6,13]$. The wordings of these two new items may still be under development, which might contribute to their unstable psychometric properties [24]. Another possible reason is that the participants in our study were institutionalized and may have had limited social lives as well as happy feelings. Thus, further studies may test these two items for people with schizophrenia who live in communities to verify our hypothesis. Items 7 and 12 were found to have higher 


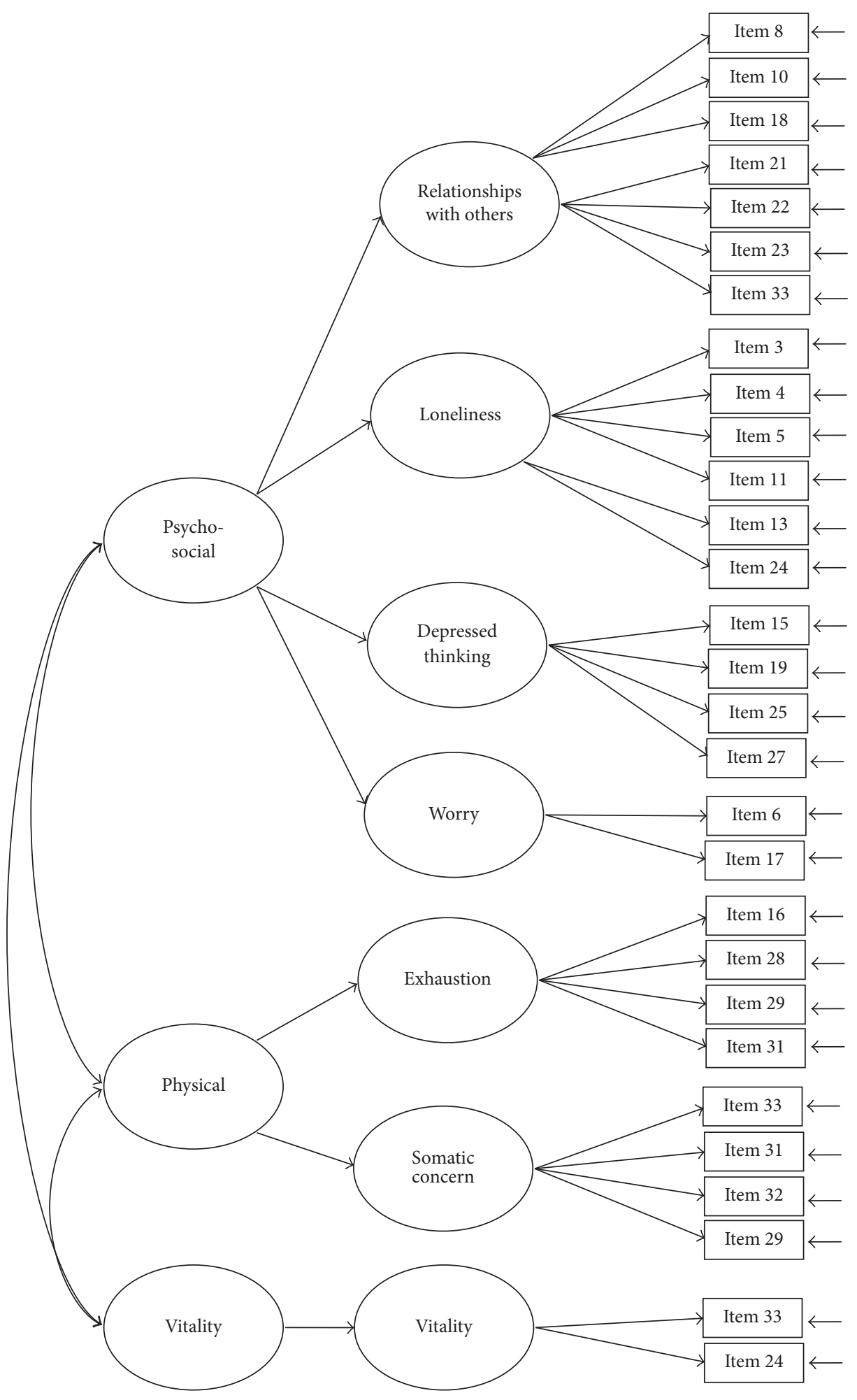

FIgURE 5: Model 5: the second-order model with 29 items. Item descriptions are in Table 3.

correlations with the total score of the motivation and energy domain in the SQLS for the UK version ( $r=0.67$ and 0.68$)$ [8] and for English-speaking Asians ( $r=0.51$ and 0.42) [11] than for the Japanese version $(r=0.39$ and 0.34$)$ [10] and for Chinese-speaking Asians ( $r=0.21$ and 0.32) [11]. Therefore, the two items may be perceived differently from the vitality construct in Asian and Western cultures $[6,12]$.

The factor structure of the SQLS/SQLS-R4 has been revised several times [9], and its psychometric properties have been tested (e.g., Wilkinson et al. [8]; Kaneda et 
TABLE 2: Model comparisons.

\begin{tabular}{|c|c|c|c|c|c|}
\hline & \multicolumn{5}{|c|}{ Model number } \\
\hline & 1 & 2 & 3 & 4 & 5 \\
\hline$\overline{\chi^{2}(d f)}$ & $860.108(494)$ & $567.519(376)$ & $544.993(374)$ & $756.583(356)$ & $675.982(368)$ \\
\hline CFI & 0.934 & 0.963 & 0.967 & 0.928 & 0.945 \\
\hline TLI & 0.929 & 0.960 & 0.964 & 0.918 & 0.939 \\
\hline RMSEA & 0.087 & 0.072 & 0.068 & 0.107 & 0.092 \\
\hline SRMR & 0.090 & 0.073 & 0.072 & 0.132 & 0.074 \\
\hline AIC & 994.108 & 685.519 & 666.993 & 914.583 & 809.982 \\
\hline ECVI & 10.041 & 6.924 & 6.737 & 9.238 & 8.182 \\
\hline
\end{tabular}

Model 1: two correlated factors (psychosocial and vitality) with 33 items.

Model 2: two correlated factors (psychosocial and vitality) with 29 items.

Model 3: three correlated factors (psychosocial, physical, and vitality) with 29 items.

Model 4: seven correlated factors (relationships with others, loneliness, exhaustion, depressed thinking, somatic concern, vitality, and worry) with 29 items.

Model 5: seven correlated factors (relationships with others, loneliness, exhaustion, depressed thinking, somatic concern, vitality, and worry) underlying three correlated dimensions (psychosocial, physical, and vitality) with 29 items.

$\mathrm{CFI}$ = comparative fit index; TLI = Tucker-Lewis index; RMSEA = root mean square error of approximation; SRMR = standardized root mean square residual; $\mathrm{AIC}=$ Akaike's information criterion; ECVI $=$ expected cross-validation index.

TABLE 3: Standardized factor loadings on SQLS-R4 with three underlying constructs.

\begin{tabular}{|c|c|c|c|c|}
\hline \multirow{2}{*}{ Item number } & \multirow{2}{*}{ Item description } & \multicolumn{3}{|c|}{ Standardized factor loadings } \\
\hline & & Psychosocial & Physical & Vitality \\
\hline 1 & Lack energy & - & - & 0.816 \\
\hline 2 & Couldn't be bothered & - & - & 0.769 \\
\hline 3 & Worry about future & 0.475 & - & - \\
\hline 4 & Lonely & 0.574 & - & - \\
\hline 5 & Hopeless & 0.654 & - & - \\
\hline 6 & Panicky & 0.632 & - & - \\
\hline 7 & Able to carry out daily activities ${ }^{\mathrm{a}}$ & - & - & - \\
\hline 8 & Took things people said the wrong way & 0.552 & - & - \\
\hline 9 & Hard to concentrate & - & 0.606 & - \\
\hline 10 & Difficult to mix with people & 0.536 & - & - \\
\hline 11 & Down & 0.753 & - & - \\
\hline 12 & Feel I can cope ${ }^{a}$ & - & - & - \\
\hline 13 & Mixed up and unsure & 0.662 & - & - \\
\hline 14 & Slept well & - & 0.261 & - \\
\hline 15 & Have mood swings & 0.769 & - & - \\
\hline 16 & Concerned wouldn't get better & - & 0.554 & - \\
\hline 17 & Worry & 0.569 & - & - \\
\hline 18 & People avoid me & 0.684 & - & - \\
\hline 19 & Upset about past & 0.537 & - & - \\
\hline 20 & Poor memory & - & 0.644 & - \\
\hline 21 & Cut off from world & 0.543 & - & - \\
\hline 22 & Uncomfortable with people & 0.740 & - & - \\
\hline 23 & Can't think clearly & 0.512 & - & - \\
\hline 24 & Upsetting thoughts & 0.766 & - & - \\
\hline 25 & Suicidal thoughts & 0.562 & - & - \\
\hline 26 & Feel happy $^{\mathrm{a}}$ & - & - & - \\
\hline 27 & Depressed & 0.740 & - & - \\
\hline 28 & Drowsy & - & 0.654 & - \\
\hline 29 & Restless & - & 0.577 & - \\
\hline 30 & Concerned about social life $\mathrm{e}^{\mathrm{a}}$ & - & - & - \\
\hline 31 & Tired & - & 0.647 & - \\
\hline 32 & Physically weak & - & 0.637 & - \\
\hline 33 & Wasn't leading normal life & 0.604 & - & - \\
\hline
\end{tabular}

All $P$ s $<0.01$

${ }^{\mathrm{a}}$ Items not in the three-correlated-factor model. 
TABLE 4: Correlations between the SQLS-R4 and the WHOQOLBREF.

\begin{tabular}{lccc}
\hline & \multicolumn{3}{c}{ SQLS-R4 } \\
& Psychosocial & Physical & Vitality \\
\hline WHOQOL-BREF & & & \\
$\quad$ Physical & -0.50 & -0.64 & -0.39 \\
Psychological & -0.41 & -0.53 & -0.26 \\
Social & -0.38 & -0.48 & -0.38 \\
Environment & -0.59 & -0.69 & -0.52 \\
\hline
\end{tabular}

All $P$ s $<0.01$.

al. [10]; and Kuo et al. [13]). Three methods have been used: concurrent validity $[6,10-12,26]$, exploratory factor analysis [8, 13], and CFA [7]. Except for the CFA, concurrent validity and exploratory factor analysis indirectly tested the construct of the SQLS-R4. Concurrent validity is used to examine the relationship between several measures [27] (e.g., the relationship between the SQLS-R4 and the WHOQOLBREF). Because the constructs of other measures (e.g., the WHOQOL-BREF) are not the same as that of the SQLSR4, the SQLS-R4 construct cannot be precisely examined using concurrent validity. Exploratory factor analysis is used to explore the possible underlying factors of one measure [28], such as the SQLS-R4; it was suitable for identifying a set of latent constructs underlying the measured items on the SQLS-R4 without an a priori hypothesis factor structure. In other words, exploratory factor analysis explores rather than examines the construct of SQLS-R4. Therefore, we suggest that it would be more appropriate to compare our results with those of Martin and Allan [7], who also used CFA to examine the factor structure of the SQLS-R4.

The main difference between the best-fit structure of the SQLS-R4 in this study and the former structure of the SQLS-R4 is one construct (physical) added to our suggested structure. Although the structure of the SQLS-R4 is designed without a physical construct $[8,9]$, the definition of QoL includes the concept of physical performance $[1,4,29]$. Therefore, the construct of physical should also be emphasized for the SQLS-R4, as Kuo et al. [13]. have recommended. The physical construct is defined as an individual's ability to perform daily activities (e.g., self-care; rest) and is related to one's physical condition [29]. Thus, some items on the SQLSR4 (e.g., physically weak; slept well; restless) are suitable for the physical construct, and they were verified in a report on health-related quality of life measures [29]. Because no published studies have examined the three-correlated-factor model of the SQLS-R4 for any Western population, we suggest that our three-correlated-factor model should be tested using Western populations to further understand the physical construct.

In addition to the CFA-based confirmation of the factor structure of the SQLS-R4, our concurrent validity test also supported the SQLS-R4 construct. Significantly moderateto-high correlations were found between the SQLS-R4 constructs and the WHOQOL-BREF constructs, except for a low correlation between the vitality construct of the SQLSR4 and the psychological construct of the WHOQOL-BREF $(r=-0.26)$. Consistent with our findings, Chou et al. [30]. also found a nonsignificant correlation between the vitality construct of the SQLS-R4 and the psychological construct of the S-QoL. The content of vitality construct items (namely, "lack energy" and "couldn't be bothered") seems to describe more about physical condition and social interaction than about mental function, which may contribute to the low correlation.

This study has several limitations. There were only 100 participants in this study, the minimum required for a reliable CFA [16, 17]. However, because the replication of a factor structure is recommended for CFA [16], our findings for the SQLS-R4 construct can be seen as a preliminary outcome for the Asian population. Future studies may conduct other CFAs based on our findings to strengthen the construct of the SQLS-R4. Second, all the participants were inpatients from one psychiatric hospital; thus, our findings may not be generalizable to people with schizophrenia and living in the community. Third, we recruited only participants with intact cognitive ability (MMSE $\geq 24$ and BPRS $<24$ ); therefore, the SQLS-R4 construct we suggested in this study cannot be generalized to those with impaired cognitive ability.

In conclusion, our results suggested a three-correlatedfactor construct of SQLS-R4 for people with schizophrenia in Taiwan. In addition, the reliability and validity were good for the SQLS-R4. However, because our participants were all institutionalized, additional studies that examine the SQLSR4 construct on outpatients with schizophrenia are needed. Moreover, comparing the SQLS-R4 construct for Asian and Western populations is also suggested.

\section{Competing Interests}

The authors declare that they have no competing interests.

\section{References}

[1] D. Basu, "Quality-of-life issues in mental health care: past, present, and future," German Journal of Psychiatry, vol. 7, no. 3, pp. 35-43, 2004.

[2] C.-Y. Lin, W.-M. Luh, C.-P. Cheng, A.-L. Yang, C.-T. Su, and H.-I. Ma, "Measurement equivalence across child self-reports and parent-proxy reports in the Chinese version of the pediatric quality of life inventory version 4.0," Child Psychiatry and Human Development, vol. 44, no. 5, pp. 583-590, 2013.

[3] A. O. Adelufosi, A. Ogunwale, O. Abayomi, and J. T. Mosanya, "Socio-demographic and clinical correlates of subjective quality of life among Nigerian outpatients with schizophrenia," Psychiatry Research, vol. 209, no. 3, pp. 320-325, 2013.

[4] The WHOQOL Group, "Study protocol for the World Health Organization project to develop a quality of life assessment instrument (WHOQOL)," Quality of Life Research, vol. 2, no. 2, pp. 153-159, 1993.

[5] J. Bobes, P. García-Portilla, P. A. Sáiz, T. Bascarán, and M. Bousoño, "Quality of life measures in schizophrenia," European Psychiatry, vol. 20, supplement 3, pp. S313-S317, 2005.

[6] P.-J. Kuo, M.-J. Chen-Sea, R.-B. Lu et al., "Validation of the Chinese version of the Schizophrenia Quality of Life Scale Revision 4 (SQLS-R4) in Taiwanese patients with schizophrenia," Quality of Life Research, vol. 16, no. 9, pp. 1533-1538, 2007. 
[7] C. R. Martin and R. Allan, "Factor structure of the Schizophrenia Quality of Life Scale Revision 4 (SQLS-R4)," Psychology, Health \& Medicine, vol. 12, no. 2, pp. 126-134, 2007.

[8] G. Wilkinson, B. Hesdon, D. Wild et al., "Self-report quality of life measure for people with schizophrenia: the SQLS," British Journal of Psychiatry, vol. 177, pp. 42-46, 2000.

[9] Oxford Outcomes Ltd, The Revised Schizophrenia Quality of Life Questionnaire (SQLS-R4): User Manual for the SQLS-R4, Oxford Outcomes, Cassington, UK, 2004.

[10] Y. Kaneda, A. Imakura, A. Fujii, and T. Ohmori, "Schizophrenia Quality of Life Scale: validation of the Japanese version," Psychiatry Research, vol. 113, no. 1-2, pp. 107-113, 2002.

[11] N. Luo, B.-K. Seng, F. Xie, S.-C. Li, and J. Thumboo, "Psychometric evaluation of the Schizophrenia Quality of Life Scale (SQLS) in English- and Chinese-speaking Asians in Singapore," Quality of Life Research, vol. 17, no. 1, pp. 115-122, 2008.

[12] N. A. Taha, M. I. Ibrahim, A. F. Rahman, A. A. Shafie, and A. H. Rahman, "Validation of the schizophrenia quality of life scale revision 4 among chronic schizophrenia patients in Malaysia," Value in Health Regional Issues, vol. 1, no. 1, pp. 82-86, 2012.

[13] P.-J. Kuo, H.-I. Ma, C.-C. Kuo, W.-C. Huang, and M.-S. Chung, "Factor analysis of the Schizophrenia Quality Of Life Scale Revision 4 (SQLS-R4) Chinese version and related factors," International Journal of Psychiatry in Clinical Practice, vol. 13, no. 4, pp. 278-284, 2009.

[14] K. A. Bollen, "Confirmatory factor analysis," in Structure Equations with Latent Variables, K. A. Bollen, Ed., pp. 226-315, John Wiley \& Sons, New York, NY, USA, 1989.

[15] American Psychological Association, Diagnostic and Statistical Manual of Mental Disorders, vol. 1, American Psychiatric Association, Arlington, Va, USA, 4th edition, 2000.

[16] P. Kline, A Psychometric Primer, Free Association Books, London, UK, 1993.

[17] J. C. Loehlin, Latent Variable Models: An Introduction to Factor, Path, and Structural Analysis, Lawrence Erlbaum Associates, Mahwah, NJ, USA, 1998.

[18] G. Yao, C.-W. Chung, C.-F. Yu, and J.-D. Wang, "Development and verification of validity and reliability of the WHOQOLBREF Taiwan version," Journal of the Formosan Medical Association, vol. 101, no. 5, pp. 342-351, 2002.

[19] Y.-C. Kao, Y.-P. Liu, M.-K. Chou, and T.-H. Cheng, "Subjective quality of life in patients with chronic schizophrenia: relationships between psychosocial and clinical characteristics," Comprehensive Psychiatry, vol. 52, no. 2, pp. 171-180, 2011.

[20] N.-W. Guo, H.-C. Liu, P.-F. Wong et al., "Chinese version and norms of the Mini-Mental State Examination," Journal of Chinese Rehabilitative Medicine, vol. 16, pp. 52-59, 1989 (Chinese).

[21] T.-J. Chang, H.-G. Hwu, and F.-W. Wei, “The inter-rater reliability of brief psychiatric rating scale (BPRS)," Chinese Society Neurology and Psychiatry Bulletin, vol. 12, pp. 29-36, 1986 (Chinese).

[22] M. A. Garcia-Barrera, R. W. Kamphaus, and D. Bandalos, "Theoretical and statistical derivation of a screener for the behavioral assessment of executive functions in children," Psychological Assessment, vol. 23, no. 1, pp. 64-79, 2011.

[23] L.-T. Hu and P. M. Bentler, "Cutoff criteria for fit indexes in covariance structure analysis: conventional criteria versus new alternatives," Structural Equation Modeling, vol. 6, no. 1, pp. 1$55,1999$.
[24] C.-Y. Lin, W.-M. Luh, C.-P. Cheng, A.-L. Yang, and H.-I. Ma, "Evaluating the wording effect and psychometric properties of the kid-kindl: using the multitrait-multimethod approach," European Journal of Psychological Assessment, vol. 30, no. 2, pp. 100-109, 2014.

[25] Y. H. Chan, "Biostatistics 104: correlational analysis," Singapore Medical Journal, vol. 44, no. 12, pp. 614-619, 2003.

[26] J. H. Kim, S. J. Yim, and S. K. Min, "The Korean version of the 4th Revision of Schizophrenia Quality of Life Scale: validation study and relationship with PANSS," Journal of Korean Neuropsychiatry Association, vol. 45, p. 401, 2006.

[27] L. G. Portney and M. P. Watkins, "Validity of measurements," in Foundations of Clinical Research: Application to Practice, L. G. Portney and M. P. Watkins, Eds., pp. 79-110, Prentice Hall Health, Upper Saddle River, NJ, USA, 2nd edition, 2000.

[28] B. G. Tabachnick and L. S. Fidell, "Principal components and factor analysis," in Using Multivariate Statistics, B. G. Tabachnick and L. S. Fidell, Eds., pp. 607-675, Pearson International, Boston, Mass, USA, 5th edition, 2007.

[29] M. J. Naughton and S. A. Shumaker, "The case for domains of function in quality of life assessment," Quality of Life Research, vol. 12, no. 1, pp. 73-80, 2003.

[30] C.-Y. Chou, M.-C. Ma, T.-T. Yang, and M.-J. Chen-Sea, "Psychometric validation of the S-QoL Chinese (Taiwan) version for patients with schizophrenia," Quality of Life Research, vol. 20, no. 5, pp. 763-767, 2011. 


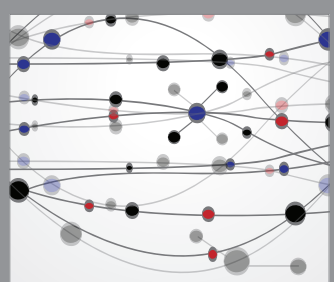

The Scientific World Journal
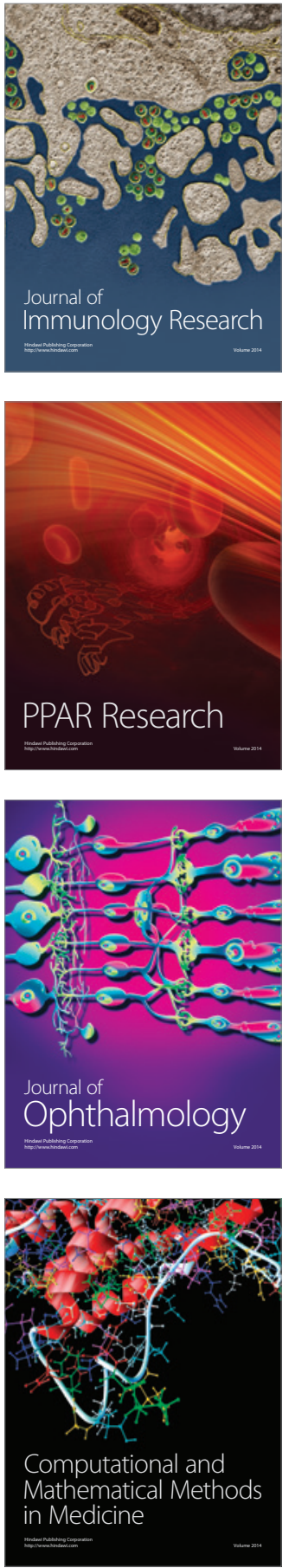

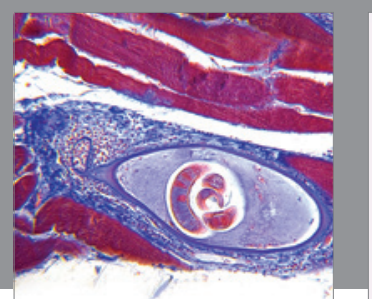

Gastroenterology Research and Practice
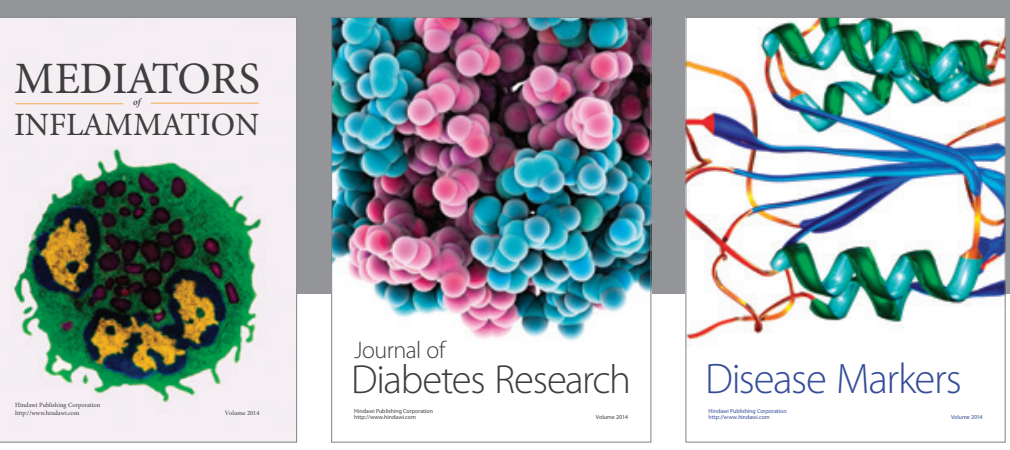

Disease Markers

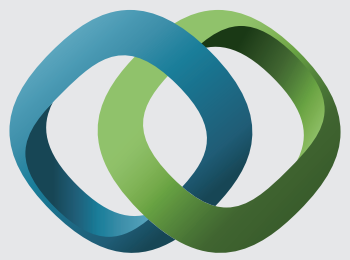

\section{Hindawi}

Submit your manuscripts at

https://www.hindawi.com
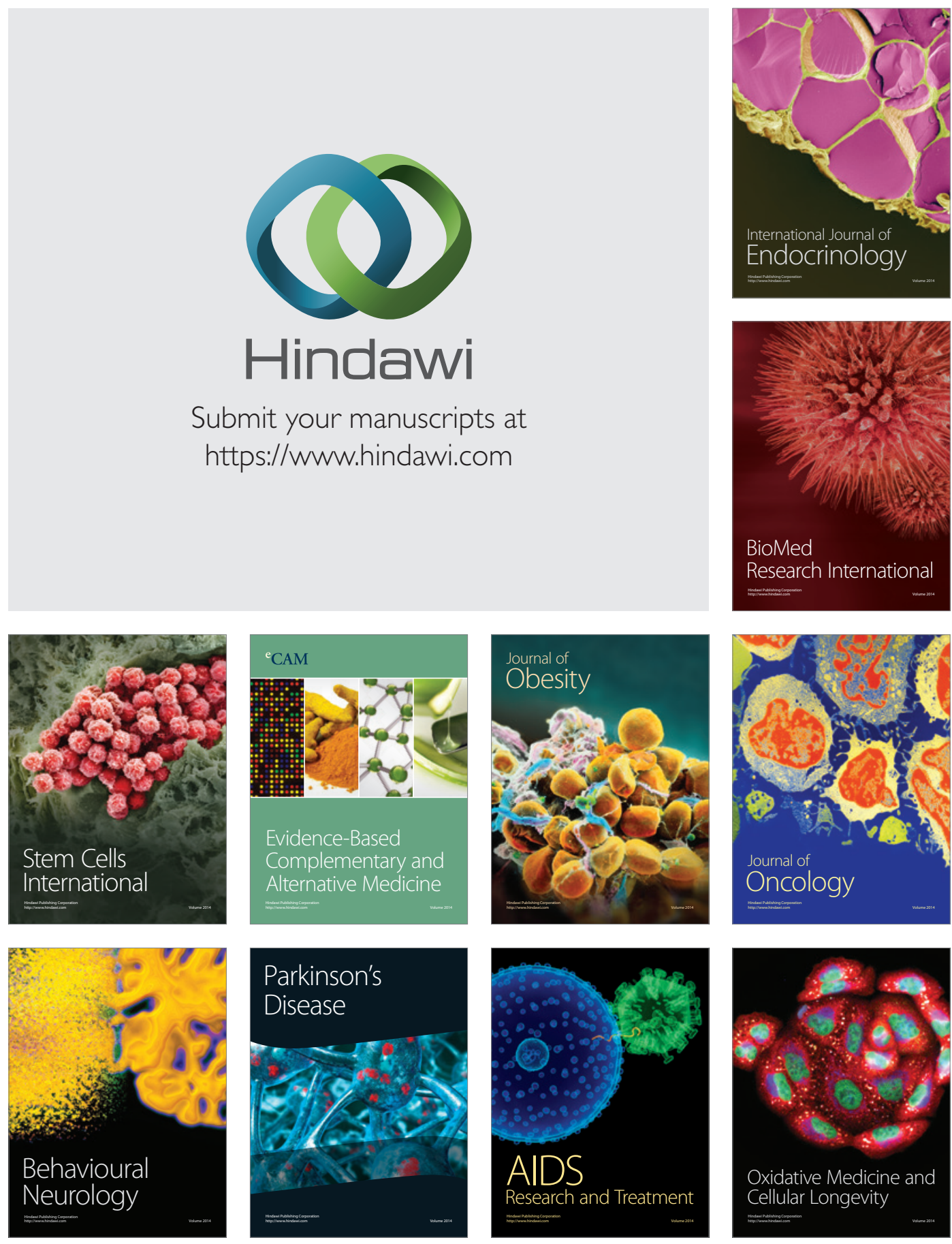